\title{
BUBBLE BURSTING AT A FREE SURFACE IN A CLOSED DOMAIN
}

\author{
LIU NIAN-NIAN, ZHANG SHUAI, and WANG SHI-PING" \\ College of Shipbuilding Engineering, Harbin Engineering University, 145, Nantong Street \\ Harbin, China \\ wangshiping@hrbeu.edu.cn*
}

Published 22 June 2016

\begin{abstract}
When a charge explodes underwater near a free surface, a bubble would be generated and the surface pushed up very high. Experiments have shown that the motion of the spike lags a lot behind the bubble motion. Many studies only focus on the nonlinear interaction between the bubble and free surface while the water waves afterward is mainly studied based on the linear theory. The nonlinear motion of the water wave after the bubble pulsation is seldom studied. In this study, we concerns the interaction between underwater explosion generated bubble and a free surface and its bursting at a free surface in a closed domain. Suppose that the fluid outside the bubble is incompressible, non-viscous and irrotational and the velocity potential satisfies the Laplace equation. Boundary integral method is used to solve the Laplace equation for the velocity potential. The bubble content is described by an adiabatic law. The whole process of the bubble motion and subsequently the water wave propagation will be simulated in this paper. Particular attention will be focused on the phenomenon of water wave propagation in a closed domain.
\end{abstract}

Keywords: Bubble dynamics; water waves; toroidal bubble; inner domain.

\section{Introduction}

Underwater explosion is the primary threat to ship structural damage, and underwater explosion bubble, as one of the main differences between the underwater explosion and the air blast, will be a fatal blow on ships. So many scholars have taken a great deal of research on underwater explosion bubble dynamics and the damage effect of bubbles on ships ${ }^{1,5}$. When the explosive package explodes, high temperature and pressure generated by the detonation products will form a bubble, if relatively closed to the free surface, the nonlinear coupling of bubble and free surface will occur intensively ${ }^{3,6}$. On one hand, the free surface makes bubble jet come up back to the free surface. On the other hand, the bubble makes free surface a giant water burial. When the water burial reach the top, it falls out by gravity effect, the uplift and fell of free surface will form the waves to the surrounding radiation, the wave has the feature of large amplitude and long cycle, which will cause the damage of surface ships and other buildings around the detonation point.

As for numerical simulation of underwater explosion bubble, since the boundary element method has unique advantages of the interface capture, the computational

This is an Open Access article published by World Scientific Publishing Company. It is distributed under the terms of the Creative Commons Attribution 3.0 (CC-BY) License. Further distribution of this work is permitted, provided the original work is properly cited. 
efficiency and accuracy, bubble dynamics simulation can often be solved by boundary element method ${ }^{2,3,4,6,7}$. Based on boundary element method, the paper established a nonlinear numerical model which the bubble and free surface interacted in a closed domain, and studied the process of bubble pulsation and the early wave generated by large equivalent kits (1000 ton) in shallow and moderate depth underwater explosion. Firstly, underwater explosion bubble pulsation and free surface rupture subjected to giant equivalents (explosive) in limited water area were studied, then the movement of bubble and water burial in the condition of vertical rigid wall and gradient rigid wall was respectively analyzed, finally the effects of water radius, water depth and water slope on bubble wave were discussed, and some disciplinarian conclusions were summarized.

\section{Basic methods}

As for underwater explosion bubble, the flow field around is assumed to be non-viscous, irrotational and incompressible ${ }^{9,10}$. The velocity potential $\Phi$ can be used to represent flow field velocity, it can be written as $\boldsymbol{U}=\nabla \Phi$, and the flow field satisfies Laplace equation.

$$
\nabla^{2} \Phi=0
$$

The velocity potential $\Phi$ of any point in the flow field can be expressed as:

$$
c \Phi\left(\boldsymbol{r}_{p}\right)=\iint_{S}\left(\frac{\partial \Phi\left(\boldsymbol{r}_{q}\right)}{\partial n_{q}} G\left(\boldsymbol{r}_{p}, \boldsymbol{r}_{q}\right)-\Phi\left(\boldsymbol{r}_{q}\right) \frac{\partial G\left(\boldsymbol{r}_{p}, \boldsymbol{r}_{q}\right)}{\partial n_{q}}\right) d S
$$

where $c$ is the solid angle, $\mathrm{r}_{p}$ and $r_{q}$ are the observing point and source point, respectively, $\mathbf{n}$ is the unit normal vector, $\mathrm{S}$ is the boundary of fluid domain, and $G\left(\boldsymbol{r}_{p}, \boldsymbol{r}_{q}\right)=\frac{1}{\left|r_{p}-r_{q}\right|}$ is Green Function in three dimensional domain.

Bubble motion satisfies the following kinematics and dynamics conditions:

$$
\begin{gathered}
\frac{d \boldsymbol{r}}{d t}=\boldsymbol{U} \\
\frac{d \Phi}{d t}=\frac{1}{2}|\boldsymbol{U}|^{2}+\frac{P_{\infty}}{\rho}-\frac{P}{\rho}-g z
\end{gathered}
$$

where $P_{\infty}$ is the pressure of infinity in $\mathrm{z}=0$ plane, $\rho$ is fluid density, and $\mathrm{g}$ is acceleration due to gravity.

When the bubble collapses, bubble jet will penetrate the bubble surface and make it a ring from a single connected domain to a double connected domain, then the velocity potential in bubble surface occurs a jump with size of $\Pi=\Phi_{i}-\Phi_{j}$ at jet shock point, which $\Phi_{i}$ and $\Phi_{j}$ are velocity potential of the impact point and the point being hit, respectively.

Wang et al. ${ }^{8}$ broke the velocity potential down as follows:

$$
\Phi_{\text {total }}=\Phi_{\text {res }}+\Phi_{\text {ring }}
$$

where $\Phi_{\text {total }}$ is the total velocity potential, $\Phi_{\text {ring }}$ is velocity potential induced by vortex rings, and $\Phi_{\text {res }}$ is the residual velocity potential. After processing of the above, the residual velocity potential in the bubble surface is continuous. Velocity potential $\Phi_{\text {ring }}$ 
and speed $\boldsymbol{U}_{\text {ring }}$ induced by vortex rings can be calculated by the integral of the velocity field originated from Biot-Savart law.

$$
\begin{gathered}
\boldsymbol{U}_{\text {ring }}\left(\boldsymbol{r}_{p}, t\right)=\frac{\Pi}{4 \pi} \oint \frac{\boldsymbol{r}_{p q} \times \mathrm{d} \boldsymbol{l}_{q}}{r_{p q}^{3}} \\
\Phi_{\text {ring }}\left(\boldsymbol{r}_{p}\right)=\frac{\Pi \Omega\left(\boldsymbol{r}_{p}\right)}{4 \pi}
\end{gathered}
$$

where $\Omega\left(\boldsymbol{r}_{p}\right)=\int_{S_{c}} \frac{\partial}{\partial n}\left(\frac{1}{r}\right) d S$ is the solid angle from the vortex ring surface $S_{c}$ to the bubble surface. The residual velocity potential of the bubble surface can be updated via the Bernoulli equation"

$$
\frac{\partial \Phi_{r e s}}{\partial t}=\frac{P_{\infty}-P}{\rho}-\boldsymbol{U}_{\text {ring }} \cdot\left(\boldsymbol{U}_{\text {ring }}+\nabla \Phi_{\text {res }}\right)-\frac{1}{2}\left|\nabla \Phi_{\text {res }}+\boldsymbol{U}_{\text {ring }}\right|^{2}-g z
$$

For the free surface, pressure $P=P_{\infty}$ in equations (2.4) and (2.8). For bubbles, pressure $P$ is the internal pressure of the bubble in equations (2.4) and (2.8). Assuming that the gas bubble generated after detonation is ideal gas which satisfies the Boyle's law:

$$
\begin{aligned}
& P=P_{c}+P_{0}\left(\frac{V_{0}}{V}\right)^{\gamma}-\sigma v \\
& P_{0}=1.39 \times 10^{5}\left(\frac{W}{V_{0}}\right)^{\gamma}
\end{aligned}
$$

where $P_{0}$ is the initial internal pressure of the bubble, $\sigma$ represents the coefficient of surface tension, $v$ is curvature, $V_{0}$ is the corresponding initial bubble volume, $W$ is the quality kits of cartridge bag, $\gamma$ is the specific heat ratio which is 1.25 for an underwater explosion bubble.

\section{Results and discussions}

\subsection{Effects of water radius on bubble wave under vertical wall}

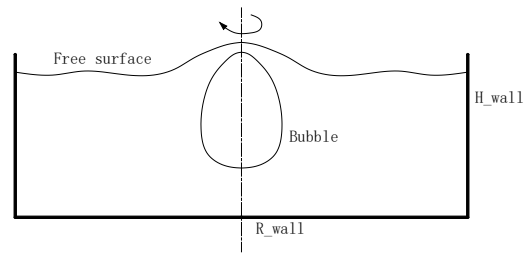

a) vertical wall



(b) inclined wall with a certain angle $\theta$

Fig. 1. Sketch map of the bubble and free surface in a limited water domain.

Fig. 1(a) shows the interaction between bubble and free surface in limited water domain under vertical wall. The bubble model in this article is axisymmetric, and the axis of symmetry is the phantom line position with the arrow indicating the direction of rotation shown in Fig. 1(a). The distance called R_wall from the axis of symmetry to the wall 
represent waters radius presented in the former content. H_wall is the depth from the initial static free surface to the bottom of the pool.

Fig. 2 illustrates the change of water burial height over time before the bubble bursts at the free surface. As shown in the figure, the smaller the radius of water, the higher the height of water burial. It is understandable that the smaller the radius of waters, the smaller the water area, then the bigger the bubble volume accounts for the proportion of waters volume and the higher the water burial. When waters radius is larger like greater than $200 \mathrm{~m}$, the height of water burial tends to the basic convergence to the explosion of infinite area.

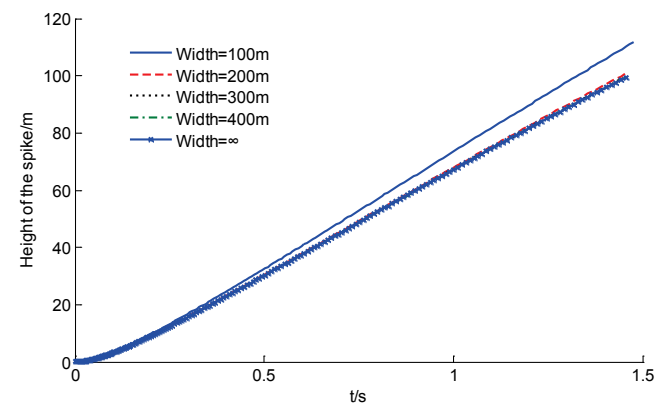

Fig. 2. The height change of water burial before bubble ruptures at the free surface

Fig. 3 represents the effects of different waters radius on the bubble pulsation in condition of one kiloton TNT explodes in depth of 50 meters. It can be seen from the figure that the smaller the waters radius, the stronger the restriction of waters area on bubble volume and bubble volume increases more slowly. As the water radius increases, the bubble volume converges to infinity radius.



Fig.3.The effect of waters radius on the change of bubble volume

\subsection{Effect of waters depth on the bubble waves}

One kiloton of TNT buried in 50 meters explodes in a limited domain at a width of $200 \mathrm{~m}$, fixing the above referred parameters and discussing the effect of waters depth on the bubble motion and waves, as shown in Fig. 4. 




Fig. 4. Effects of depth of the water on the height of water column

Fig. 4 shows effects of depth of the water on the height of water column in different pool depth under the condition of $400 \mathrm{~m}$ radius of the pool. As can be seen from fig. 4 , when the pool depth keeps $100 \mathrm{~m}$, the movement of water column appeared very different for the pool opened at the end of the explosion. When the water depth is deep, the height of water burial converges to infinity radius.

When the explosion occurred in closed domain, people will care about the climbing height of shore water along the wall in the process of free surface movement. Fig. 5 shows the climbing height of waters along the wall in different pools depth in condition of $400 \mathrm{~m}$ waters radius and one kiloton of TNT bursts in 50 meters depth. As shown in fig.5, when the water depth is shallow $(100 \mathrm{~m})$, the pool will reveal the bottom in this case, and the water will climb intensively up to the highest position $2.256 \mathrm{~m}$ at the nearly moment of $t=10.96 \mathrm{~s}$. With the increase of water depth, the climbing height of the water along the wall will convergence to infinite water depth.

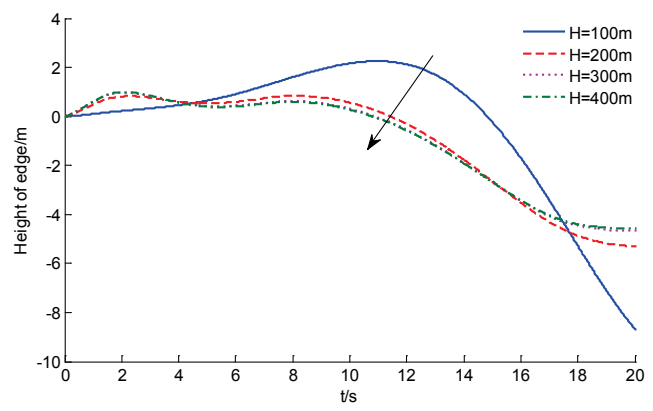

Fig. 5. Effects of water depth on the climbing height of shore water

\subsection{Effects of the bottom slope on bubble waves}

When underwater explosion occurs, the sea floor is not necessarily level, especially in the situation for shallow water. Under the condition of shallow water, the bottom of the sea will be at an angle to the shore. This section will discuss the effect of such an angle on bubbles wave. The initial state of this part is still that one kiloton TNT explodes at 50m depth and center depth is 200 meters as well as the free surface radius is 200 meters. The interaction of bubble and free surface under the condition of limited wall area with a 
certain angle is shown in Fig. 1(b). The wall angle is the angle between the pool wall and the horizontal plane, as shown $\theta$ in Fig. 1(b).

Fig. 6 shows the change curve of water burial height in different water surface width, among them the surface width radius are respectively $200 \mathrm{~m}, 300 \mathrm{~m}, 400 \mathrm{~m}, 600 \mathrm{~m}$, and corresponding wall angle are respectively $45^{\circ}, 33.7^{\circ}, 26.7^{\circ}, 18.4^{\circ}$. As can be seen from Fig. 6, when the surface width radius is $200 \mathrm{~m}$, water burial height is relative high in the early, but it will reach the minimum value while the time is greater than $3 \mathrm{~s}$, this is relevant to the narrow fluid region. As shown in Fig. 6, as the surface width widens, water burial height converges to the limit of $0^{\circ}$.



Fig. 6. The change curve of water burial height in different water surface width

\section{Conclusions}

In this paper, based on ideal incompressible potential flow theory, an axisymmetric boundary element model of underwater explosion bubble interacting with free surface and rigid wall was presented. The movement of bubbles and water burial in the condition of vertical and slope rigid wall was studied, then the effects of water radius, water depth and water slope on bubble wave were discussed. Through the analysis, following main conclusions have been drawn:

(1) Via calculating the energy change of bubble over time in all progress which bubble raptures in the surface and then forms bubble waves in vertical wall, it is found that the energy keeps the balance and the effectiveness of numerical methods to calculate the inner problem of explosion bubble waves in this article.

(2) As for big charge underwater explosion, if the water depth is very shallow, explosion can make the pool reveal the bottom easily, and then the water column height generated in this case is low.

(3) While the waters in different radius, different depths and different slope conditions, the calculation results respectively converge to infinite waters radius, infinite waters depth and the pool slope tend to $0^{\circ}$. 


\section{Acknowledgement}

The authors would like to acknowledge the support of the National Program for Support of National Natural Science Foundation of China (No. 11202057) and Key Laboratory of coastal and offshore engineering of Dalian University of Technology (No. LP1408).

\section{References}

1. Zhang X C 2008 J. Ship Mech. 12490.

2. Rungsiyaphornrat S, Klaseboer E, Khoo B C, Yeo K S 2003 Comp. Fluids 321049.

3. Wang Q X, Blake J R 2010 J. Fluid Mech. 659 191 1-34.

4. Klaseboer E, Hung K C, Wang C, Wang C W, Khoo B C, Boyce P, Debono S, Charlier H 2005 J. Fluid. Mech. 537387.

5. Liu C R, Zhou X C, Huhe A D 1997 J. Hydr. 12315.

6. Pozrikidis C 2004 Eng. Anal. Bound. Elem. 28315.

7. Qi D M, Lu C J, He Y S 2000 Chin Quart. Mech. 2116.

8. Wang Q X, Yeo K S, Khoo B C, Lam K Y 1996 Comp. Fluids 25607.

9. Zhang Y L, Yeo K S, Khoo B C, Wang C 2001 J. Comp. Phys. 16336.

10. Zhang A M, Yao X L 2008 Acta Physica Sinica 57339. 